\title{
Bruceanol D
}

National Cancer Institute

\section{Source}

National Cancer Institute. BruceanolD. NCI Thesaurus. Code C1535.

A quassinoid phytochemical isolated form the plant Brucea antidysenterica with potential antineoplastic activity. ( $\mathrm{NCl} 04)$ 